\title{
Beyond bulk entrainment and detrainment rates: $A$ new framework for diagnosing mixing in cumulus convection
}

\section{Citation}

Nie, Ji, and Zhiming Kuang. 2012. "Beyond Bulk Entrainment and Detrainment Rates: A New Framework for Diagnosing Mixing in Cumulus Convection: MAPPING FRAMEWORK FOR CONVECTIVE MIXING." Geophysical Research Letters39 (21): n/a-n/a. https:// doi.org/10.1029/2012GL053992.

\section{Permanent link}

http://nrs.harvard.edu/urn-3:HUL.InstRepos:41534502

\section{Terms of Use}

This article was downloaded from Harvard University's DASH repository, and is made available under the terms and conditions applicable to Other Posted Material, as set forth at http:// nrs.harvard.edu/urn-3:HUL.InstRepos:dash.current.terms-of-use\#LAA

\section{Share Your Story}

The Harvard community has made this article openly available.

Please share how this access benefits you. Submit a story.

\section{Accessibility}




\title{
Beyond bulk entrainment and detrainment rates: A new framework for diagnosing mixing in cumulus convection
}

\author{
$\mathrm{Ji} \mathrm{Nie}^{1}$ and Zhiming Kuang ${ }^{2}$ \\ Received 27 September 2012; revised 5 October 2012; accepted 5 October 2012; published 1 November 2012.
}

[1] A new framework for diagnosing mixing in cumulus convection is proposed, where the vertical evolution of the cloud mass flux distribution as a function of given parcel properties is viewed as a mapping process. Matrices that characterize this mapping process are constructed using a Lagrangian particle dispersion model embedded in a Large Eddy Simulation. Extending previous use of LES-diagnosed bulk entrainment and detrainment rates to test bulk plume models, the construction of these mapping matrices from LES provides a reference, against which multiple-parcel models can be compared. The framework is further applied to diagnose the response of shallow cumuli to a small temperature perturbation. Citation: Nie, J., and Z. Kuang (2012), Beyond bulk entrainment and detrainment rates: A new framework for diagnosing mixing in cumulus convection, Geophys. Res. Lett., 39, L21803, doi:10.1029/2012GL053992.

\section{Introduction}

[2] Despite the success of bulk entraining/detraining plume models [e.g., Siebesma and Cuijpers, 1995; Bretherton et al., 2004] in reproducing the domain averaged heating and moistening rates of shallow cumulus convection, there have been increasing efforts on multiple-parcel/plume models [e.g., Emanuel, 1991; Neggers et al., 2002; Cheinet, 2004; Nie and Kuang, 2012]. The multiple-parcel/plume models (simply called multi-parcel models hereafter) can potentially account for in-cloud turbulent fluxes. More importantly, they naturally predict the variability of cloudy updrafts. Representing the heterogeneity within the updrafts can be important for microphysics and chemistry, which involve highly non-linear reactions or microphysical processes. Thus, the multi-parcel models are more versatile and can be a useful extension of the bulk plume models.

[3] A key aspect of the multi-parcel models is the representation of turbulent mixing among cloudy updrafts and between cloudy updrafts and their environment. Detailed numerical simulations such as Large Eddy Simulations (LES) can potentially be used to constrain such representations, provided that the LES results are diagnosed properly in the framework of a given conceptual model or parameterization scheme. In the framework of a bulk plume model,

\footnotetext{
${ }^{1}$ Department of Earth and Planetary Sciences, Harvard University, Cambridge, Massachusetts, USA.

${ }^{2}$ Department of Earth and Planetary Sciences, School of Engineering and Applied Sciences, Harvard University, Cambridge, Massachusetts, USA.

Corresponding author: Ji Nie, Department of Earth and Planetary Sciences, Harvard University, Cambridge, MA 02138, USA. (jinie@fas.harvard.edu)

C2012. American Geophysical Union. All Rights Reserved. 0094-8276/12/2012GL053992
}

such turbulent mixing is characterized by the bulk entrainment/detrainment rates, and Siebesma and Cuijpers [1995] pioneered an analysis of LES results to evaluate entrainment/detrainment rates used in bulk plume models. Here, we propose a diagnostic framework that extends the analysis of Siebesma and Cuijpers [1995] to multi-parcel models. After introducing our framework, we will apply it to a shallow convection case and compare the results to those from two multi-parcel models. We will then apply it to probe the response of shallow convection to a large-scale temperature anomaly [Kuang, 2010; Tulich and Mapes, 2010; Nie and Kuang, 2012].

\section{The Mapping Diagnostic Framework}

[4] We shall assume that transports by cloudy updrafts and the corresponding environmental subsidence dominate the total convective transports, which is reasonable for shallow cumulus convection [Siebesma and Cuijpers, 1995]. We define cloudy updrafts as grids with liquid water content greater than $0.01 \mathrm{~g} \mathrm{~kg}^{-1}$ and vertical velocity $w$ greater than $1 \mathrm{~m} \mathrm{~s}^{-1}$. Conclusions here are not sensitive to this definition. The cloudy updraft mass flux can be binned on a given parcel property $\phi$ (such as total water $q_{t}$ or liquid water potential temperature $\theta_{l}$ ) as a distribution function $m(\phi)$. With the assumption that the environment is homogenous, the total convective flux of $\phi$ is

$$
F(\phi)=\int m(\phi)\left(\phi-\phi_{e n v}\right) d \phi
$$

where $\phi_{\text {env }}$ is the environmental $\phi$. Instead of assuming an uniform $\phi$ in cloudy updrafts as in bulk plume models, the mass flux distribution $m(\phi)$ provides a characterization of the in-cloud variations of $\phi$. The environment is assumed to be homogeneous here to simplify the analysis. The mapping framework is sufficiently general, if needed, to account for heterogeneity in the environment as well.

[5] We shall view the evolution of $m(\phi)$ with height as a mapping process. From a level $\mathrm{n}$ to level $\mathrm{n}+1$, the function that maps $m_{n}(\phi)$ onto $m_{n+1}\left(\phi^{\prime}\right)$ (Prime indicates the higher level.) describes the result of turbulent mixing of cloudy updrafts and the environment from the perspective of property $\phi$.

[6] Next we introduce how to construct the mapping function in a discretized form using the LPDM (Lagrangian particle dispersion model) [Weil et al., 2004]. Figure 1 is a diagram of the process, where each box represents a cloudy updraft grid and each arrow represents a trajectory of a Lagrangian particle that links two grids at different heights. Starting from a grid, there are $N$ Lagrangian particles (in the diagram, 3 particles are plotted as an illustration.) that are advected by LES winds and are dispersed with time. It is 


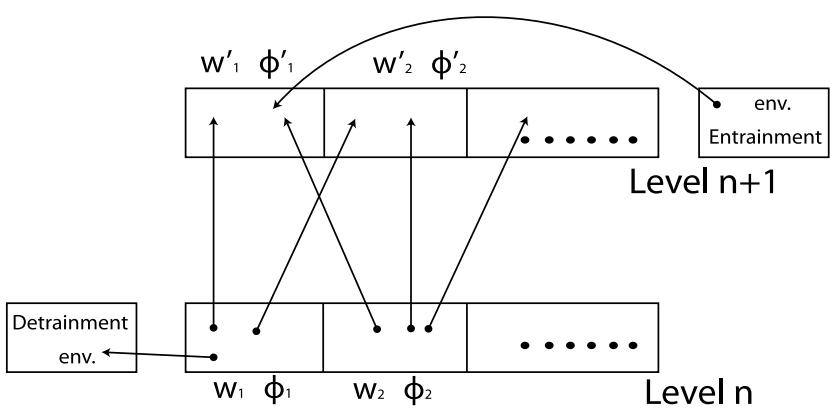

Figure 1. A diagram of the mapping process. See text for details.

assumed that each particle represents $1 / N$ of the grid's mass. For a cloudy updraft, for example, grid "1", a subset of the particles may leave the cloud and enter the environment, which represents the detrained mass flux. We bin these particles on $\phi$ to define a diagonal matrix $D(\phi, \phi)$ that describes the fractional detrainment rate per unit mass flux as a function of $\phi$. For the particles that rise to level $n+1$ inside a cloud, due to turbulent mixing, the trajectories are dispersed to different grids, for example, grids with $\phi_{1}^{\prime}$ and $\phi_{2}^{\prime}$. Each of these trajectories connects some portion of the mass flux with certain $\phi$ at level $\mathrm{n}$ and some portion of the mass flux with certain $\phi^{\prime}$ at level $\mathrm{n}+1$. These trajectories are binned into the mapping matrix $A\left(\phi^{\prime}, \phi\right)$ and normalized by the non-detrained mass flux. For a grid at level $n+1$, not all of its particles can be tracked back to cloudy updrafts at level $\mathrm{n}$. Some are entrained from the environment. Similar to $D$, a diagonal entrainment rate matrix $E\left(\phi^{\prime}, \phi^{\prime}\right)$ is defined as the ratio of the entrained mass flux to the non-entrained mass flux.

[7] Since we are interested in the local mixing processes as cloudy updrafts move from one level to another, we track each particle only within the time interval between $t_{0}-\tau$ and $t_{0}+\tau$, where $t_{0}$ is the time the particle crosses from level $\mathrm{n}$ to level $\mathrm{n}+1$. For a vertical spacing of $50 \mathrm{~m}$ used in this study (section 3), we use a $\tau$ of $50 \mathrm{~s}$, which is the time that the slowest updrafts (from our definition of $w>=1 \mathrm{~ms}^{-1}$ ) take to traverse this vertical spacing. If a parcel leaves and re-enters cloudy updrafts between $t_{0}-\tau$ and $t_{0}+\tau$, its departure and reentrance are not considered as detrainment and entrainment events. The results here are not sensitive for $\tau \in[40 \mathrm{~s}, 100 \mathrm{~s}]$.

[8] We do the tracking for all particles in cloudy updrafts so that the mapping from $m_{n}(\phi)$ to $m_{n+1}\left(\phi^{\prime}\right)$ reads

$$
m_{n+1}\left(\phi^{\prime}\right)=\left(I+E\left(\phi^{\prime}, \phi^{\prime}\right)\right) A\left(\phi^{\prime}, \phi\right)(I-D(\phi, \phi)) m_{n}(\phi),
$$

where $I$ is the identity matrix. In equation (2), the mapping process is naturally separated into three components with clear physical meaning; the detrainment rate matrix $D(\phi, \phi)$, the entrainment rate matrix $E\left(\phi^{\prime}, \phi^{\prime}\right)$ and matrix $A\left(\phi^{\prime}, \phi\right)$ that maps the non-detrained mass flux to the non-entrained mass flux. Equation (2) can be applied recursively over multiple levels. Knowing the mass flux distribution on one level (e.g., cloud base) and the mapping matrices upward from that level, the mass flux distributions on all levels above can be determined and so can the flux $F(\phi)$. We note that Kuang and Bretherton [2006] also binned mass flux in terms of moist static energy and examined how its distribution varies with height in the framework of Arakawa and Schubert [1974]. Our analysis here, through the use of a LPDM, significantly extends that study in that bins at different heights are connected by actual LES flow trajectories, instead of a hypothesized entraining plume model.

[9] The more conventional concepts of bulk entrainment and detrainment rates can be viewed as the mapping functions with only one bin for the cloudy updrafts and one bin for the environment, i.e., the top-hat approximation. In the context of such a bulk plume model, entrainment and detrainment rates obtained from tracking the movement of air masses across the cloud-environment interface (as in, e.g., the work of Romps [2010] and Dawe and Austin [2011a, 2011b] and with the Lagrangian particles as done here and contemporaneously by Yeo and Romps [2012]) cannot describe the exchange of thermodynamic properties and tracers between the two bins. This is simply because bins in the bulk plume model are too coarse and miss the significant heterogeneity within a bin: air entrained from the environment or detrained from the cloud does not have the mean properties of its originating bin. Here, we use bins that are fine enough so that heterogeneity within each bin is insignificant in terms of $\phi$ and variables that covary with $\phi$. In this paper, we use only one bin for the environment, but the mapping framework allows for multiple bins for the environment as well. Entrainment and detrainment rates obtained from tracking the movement of air masses (done here through the LPDM) can now describe the exchanges of $\phi$ (and other properties that are sufficiently uniform within each bin), because they are given as functions of $\phi$. Therefore, the mapping functions provide a more complete description of the mixing process and are a useful extension to the bulk entrainment/detrainment rates of the bulk plume model.

[10] To better understand and parameterize convection, it is valuable to examine how convection responds to changes in its large-scale environment. We shall focus on the response to small perturbations, for which the changes of mass flux distribution can be separated into four terms by applying the chain rule on equation (2);

$$
\begin{aligned}
\delta m_{n+1}= & (I+E) A(I-D) \delta m_{n}-(I+E) A \delta D m_{n} \\
& +(I+E) \delta A(I-D) m_{n}+\delta E A(I-D) m_{n}
\end{aligned}
$$

The first term is associated with $\delta m_{n}$, the inputs at level $\mathrm{n}$, keeping the mapping matrices unchanged. We shall call it the inherited term. The second to the fourth terms are changes of the mass flux distribution due to changes in the detrainment rate $D$, the mapping matrix $A$, and the entrainment rate $E$, respectively.

[11] The coordinate $\phi$ need not be a single variable. Mapping functions for joint mass flux distributions in terms of multiple variables (e.g., $q_{t}$ and $w$ ) can in principle be constructed as well. The vertical distance over which the mapping matrices are constructed can be coarser than the LES vertical resolution based on the problem of interest. While we focus on shallow convection in this paper, the analysis framework is also applicable to deep convection.

\section{Experiments Design}

[12] In this and next sections, we will apply the mapping framework to a case of shallow convection and its response to a small temperature perturbation. The case that we use is 
(a)

(c)

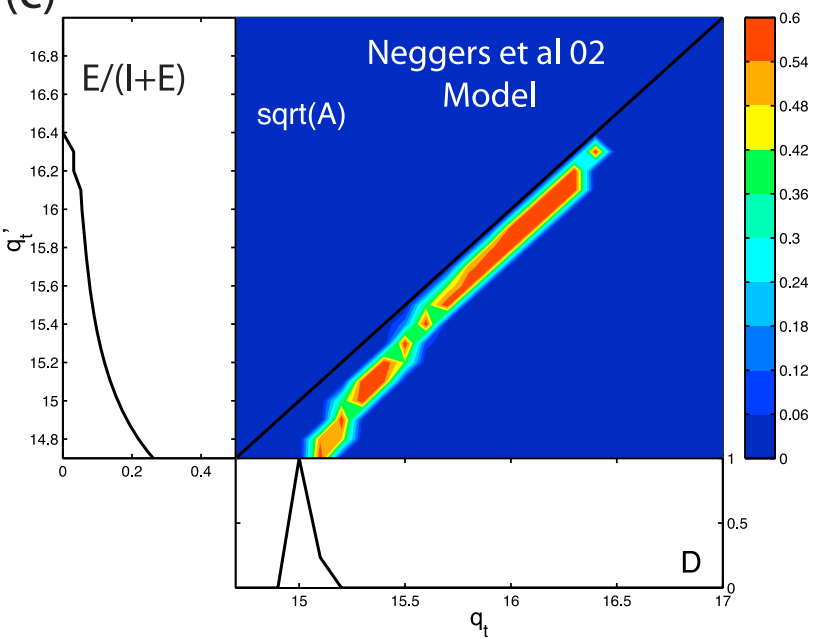

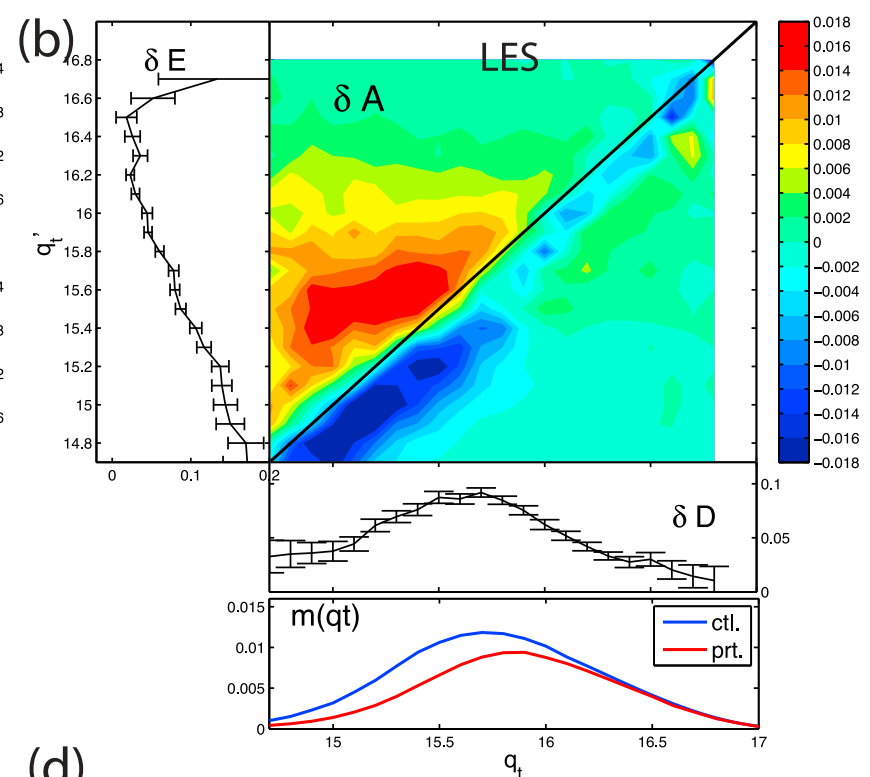

(d)

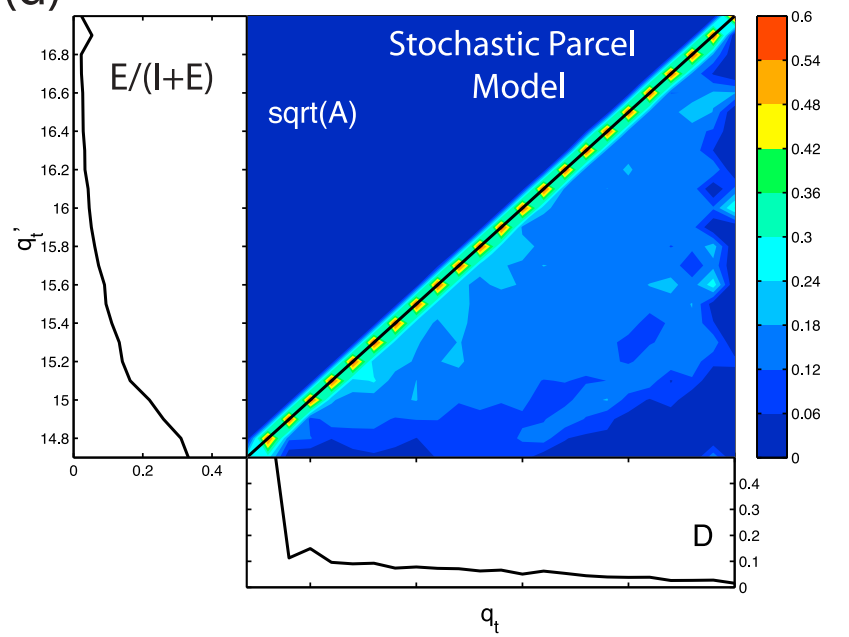

Figure 2. (a) The control run mapping matrices from $975 \mathrm{~m}$ to $1025 \mathrm{~m}$ using $q_{t}$ as the coordinate. The lowest panel shows the distribution $m\left(q_{t}\right)$ at the $975 \mathrm{~m}$ level. The diagonal matrices $D$ and $E /(I+E)$ are plot as function of $q_{t}$. The color map shows the matrix sqrt(A) (unitless) with the 1-to-1 line (black solid) superimposed. (b) Same as Figure 2a but for the differences in the mapping matrices between the perturbed runs and the control runs. The color map shows $\delta A$.The error bars show the estimated uncertainties using the jackknife method. Mapping matrices of the control runs for (c) the Neggers Model and (d) the stochastic parcel model.

the undisturbed Barbados Oceanographic and Meteorological Experiment (BOMEX) [Holland and Rasmusson, 1973]. The forcing and other settings are the same as the intercomparison study of BOMEX described in Siebesma et al. [2003].

[13] The numerical simulation is done with the System for Atmospheric Modeling (SAM) model in a domain of $6 \mathrm{~km}$ $(\mathrm{x}) \times 6 \mathrm{~km}(\mathrm{y}) \times 3 \mathrm{~km}(\mathrm{z})$. The LPDM is similar to that in Weil et al. [2004] and Heus et al. [2008] except that subgrid diffusion is not included. Heus et al. [2008] showed that including subgrid diffusion has little impact on the particles in the cloud layer. Properties of a particle inside an LES grid are set to the grid values with no interpolation. Using linear interpolation, as in Weil et al. [2004] and Heus et al. [2008], has little effects on the results presented here. The two choices (no interpolation and linear interpolation) are consistent with first- and second-order advection, respectively, and are expected to bracket the actual order of SAM's advection. To better resolve the mixing process, we use a resolution of $25 \mathrm{~m}$ in all directions, and release 2 Lagrangian particles in each grid. The LES output is coarsened to a resolution of $100 \mathrm{~m} \times 100 \mathrm{~m} \times 50 \mathrm{~m}$, so there are 64 particles in a coarsened grid, sufficient to generate robust statistics. To test the robustness of the results with respect to resolution and the number of particles, we performed the same analysis on simulations with a resolution of $100 \mathrm{~m} \times$ $100 \mathrm{~m} \times 50 \mathrm{~m}$ with 64 or 27 particles released in each grid, and the results are similar to those reported here.

[14] The model is run for 6 hours and the first 3 hours are discarded as spinup. Between hours 3 to 6 , restarts are saved every 5 minutes. The model is run for half an hour with the embedded LPDM turned on using these restarts with and without a temperature perturbation $\delta T=0.25 \mathrm{~K} \exp \left[-\frac{1}{2}\left(\frac{z-1025 \mathrm{~m}}{100 \mathrm{~m}}\right)^{2}\right] \quad($ shown in Figure 3f) introduced. The results in the next section are averaged 

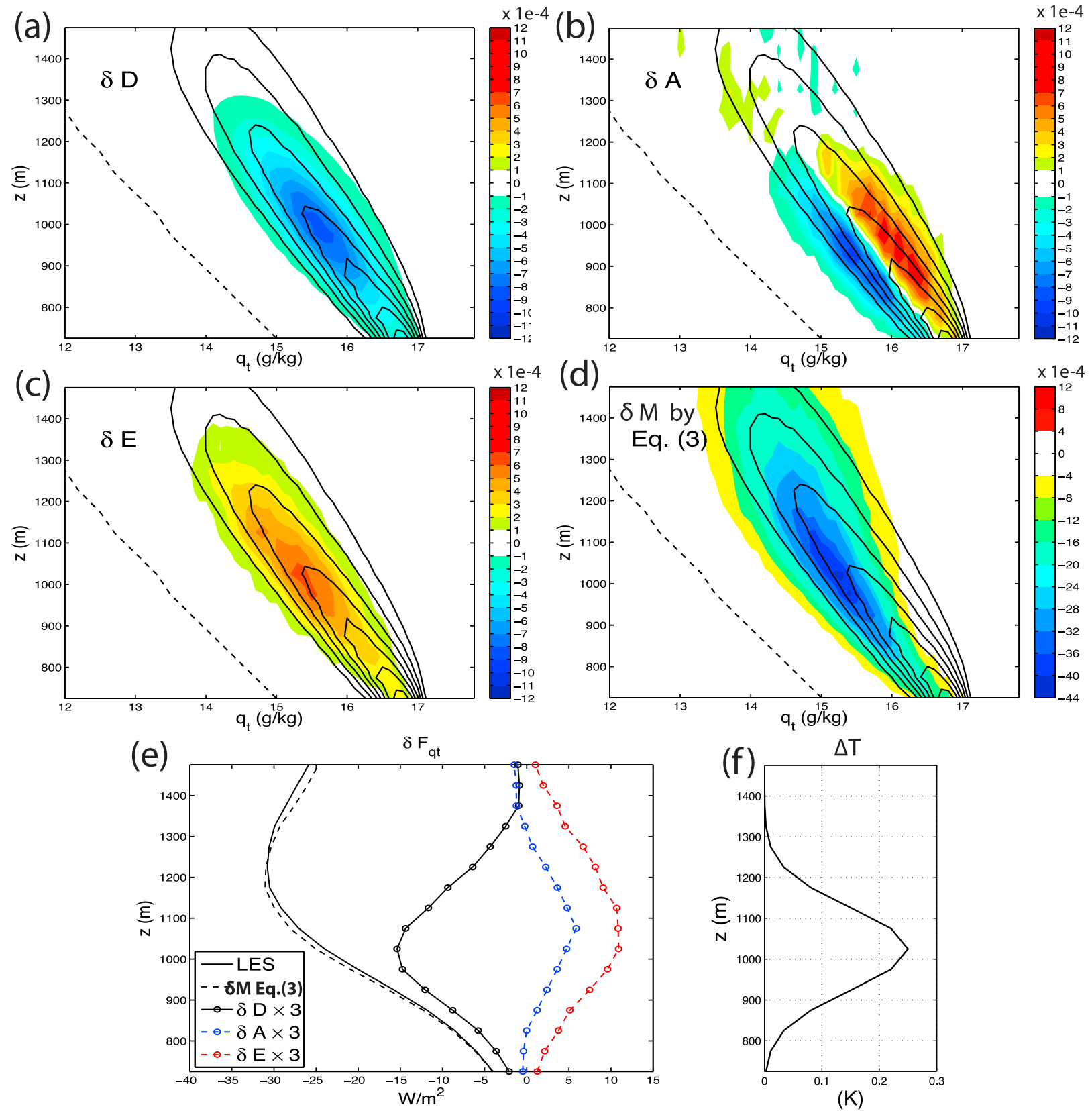

Figure 3. $(\mathrm{a}-\mathrm{c})$ The color contours are the local sources terms in equation (3). ( $\mathrm{d}$ The color contours are the total $\delta m$ computed from equation (3). In Figures 3a-3d, the color contours are in units of $\mathrm{kg} \mathrm{m}^{-2} \mathrm{~s}^{-1}$ per g kg${ }^{-1}$ (i.e., normalized by the bin interval). (e) The $\delta F_{q t}$ corresponding to mass flux changes shown in Figures 3a-3d, together with $\delta F_{q t}$ from the LES. In Figure $3 \mathrm{e}$ the $\delta D, \delta A$ and $\delta E$ terms are multiplied by a factor of 3 for better illustration. (f) Introduced temperature perturbation.

over 150 ensemble members and they are statistically robust.

[15] We shall use $q_{t}$, which is conserved in shallow nonprecipitating convection, as the coordinate. While $\theta_{l}$ is also conserved, the introduced temperature perturbation changes the environmental $\theta_{l}$ in the perturbed runs, which causes additional complications when $\theta_{l}$ is used as the coordinate.

\section{Results}

[16] The control run mapping matrices from the $975 \mathrm{~m}$ height to the $1025 \mathrm{~m}$ height (chosen to be close to the center of the introduced perturbation) is shown in Figure 2a. The lowest panel is the mass flux distribution at $975 \mathrm{~m}\left(m_{n}\right.$ in equation (2)). The detrainment matrix D shows that about $10 \%$ to $30 \%$ of the mass flux is detrained over this $50 \mathrm{~m}$ layer (about 2-6 per km, comparable to the values from Siebesma and Cuijpers [1995]). The detrainment rate is greater at lower $q_{t}$, indicating that updrafts with lower $q_{t}$ detrain more strongly. The color map shows the matrix A (we plot sqrt(A) instead of A for a better illustration), where each column indicates how one unit of non-detrained mass flux on level $n$ with a certain value of $q_{t}$ is distributed on level $\mathrm{n}+1$ (in terms of $q_{t}$ ). Without any mixing, nonzero values of $A$ will 
lie on the 1-to-1 line, and the values will deviate from unity only because of vertical acceleration or deceleration. Mixing with environmental air decreases updrafts' $q_{t}$. As a result, the peak values of $A$ are slightly below the 1-to-1 line. Besides mixing with the environment, in-cloud mixing among the cloudy updrafts homogenizes their properties, shrinking the range of $q_{t}$. The regions that are above the 1-to-1 line are the results of in-cloud mixing. The entrainment rate $E$ has the same trend as $D$, with values decreasing as $q_{t}$ increases, as grids with large amounts of entrained air have lower $q_{t}$. Note that in Figure 2, we plot $E /(I+E)$ which is the ratio of entrained air mass to the total mass after the entrainment, which, like values in D, is always less than 1. For the other layers not shown here, the general dependence of $\mathrm{D}$ and $\mathrm{E}$ on $q_{t}$ is similar.

[17] The mapping matrices set a reference against which multi-parcel models can be compared. The mapping matrices A, D, E of a bulk plume models are all single numbers (or a single point in Figure 2a). The mapping matrices of two types of multi-parcel models are shown here for comparison with the LES results. One is the model developed in Neggers et al. [2002] (Figure 2c), where there is only detrainment for updrafts with the lowest $q_{t}$, and the entrainment rate increases as $q_{t}$ decreases. The mapping matrix $\mathrm{A}$ is a single line slightly below the 1-to-1 line. This line is further away from the 1-to-1 line at low $q_{t}$ values. This feature results from the assumption in the Neggers et al. model that entrainment rate is inversely proportional to $w$, which is well correlated with $q_{t}$. Another model is the stochastic parcel model [Nie and Kuang, 2012; Romps and Kuang, 2010] (Figure 2d). For the mapping matrix A, there is a strong peak on the 1-to-1 line, representing unmixed parcels. There is also a broad triangular region below, which is due to stochastic entrainment. The detrainment rate is non-zero over all $q_{t}$ values and decrease as $q_{t}$ increases. As is apparent from Figure 2, the mapping matrices from the muliti-parcel models differ substantially from those deduced from the LES. A prominent difference is that because in-cloud mixing is not included in the multi-parcel models, their mapping matrices A are all zero above the 1-to-1 line. This discrepancy is also pointed out when Nie and Kuang [2012] used their model to reproduce the updraft statistics using the tracer encoding method of Romps and Kuang [2010]. Addition of this missing component should be considered for future models.

[18] The difference of the matrices between the control runs and the perturbed runs, averaged over the 150 pairs of ensemble members, are shown in Figure $2 b$. The error bars for $\delta D$ and $\delta E$ are standard deviation estimated by a jackknife method that leaves out one pair of runs at a time. As expected, with the temperature perturbation, more mass flux is detrained (generally positive $\delta D$ ). The reason for the variations of $\delta D$ with $q_{t}$ is not clear and left to future study. For $\delta A$, the pattern is a general upward shift to higher $q_{t}$ at level $\mathrm{n}+1$ because the warm anomaly tends to preferentially eliminate mixtures with low $q_{t}$ (less active) [Nie and Kuang, 2012]. The positive $\delta E$ shows more entrainment of environmental air in response to the positive temperature perturbation. A possible explanation of the enhanced entrainment is that the warm anomaly acts as a buoyancy barrier and slows down the updrafts, so that there is more time for entrainment per unit distance [Neggers et al., 2002], although more work is needed to test this idea.
We note that this dependence, if existent, is not strong enough to explain the in-cloud heterogeneity in the way hypothesized in Neggers et al. [2002], and in-cloud heterogeneity is mostly caused by stochastic entrainment, as shown in Romps and Kuang [2010].

[19] The contribution of each component can be estimated using equation (3) for all levels. The $\delta D, \delta A$ and $\delta E$ terms in equation (3) are local (in z) sources to the $\delta m_{n}+1$. The inherited term, although dominating the equation, is simply the accumulated impacts from levels below. The local source terms are shown in Figures $3 a-3 c$, together with the control run mass flux distribution (background black contour) and control environmental $q_{t}$ (the far left black dashed line). The $\delta D$ term is strongly negative, and contributes to the decrease of mass flux. The $\delta E$ term is positive, but to a lesser extent. The $\delta A$ term shows a shift from the left to the right of the ridge of the control mass flux distribution. It leads to a stronger reduction in mass flux at lower $q_{t}$ and the increase of updraft mean $q_{t}$ in the perturbed cases. We apply equation (3) recursively starting from a level slightly above cloud base (675 $\mathrm{m}$ height) upward with the local source terms added in each layer. The total $\delta m$ computed from the chain rule (equation (3)), shown in Figure $3 \mathrm{~d}$, is very close to the $\delta m$ directly sampled from LES, a validation of the chain rule separation. In Figure 3e, effects of these terms are summarized in terms of $\delta F_{q_{t}}$ (using equation (1)). It shows that the local source terms are mostly confined in the perturbed layer. Although $\delta m$ is still large above the perturbed layer, it is mostly accumulated over the perturbed layers below.

\section{Summary and Discussions}

[20] A new framework for diagnosing mixing in cumulus convection is proposed, where the vertical evolution of the cloud mass flux distribution as a function of given parcel properties is viewed as a mapping process. By analyzing the trajectories of LPDM embedded in an LES, the matrices that characterize this mapping process are constructed. The framework is applied to the shallow cumulus convection case of BOMEX. The mapping matrices of two multi-parcel models are compared with those from the LES, showing major discrepancies. Changes in the mapping matrices in response to a small temperature perturbation in the cloud layer are used to better understand such responses, yielding conclusions similar to those reached in Nie and Kuang [2012] using a different analysis. We believe that these mapping functions provide a more complete description of the mixing process that helps to better connect LES with convective parameterization, and are a useful extension to the bulk entrainment/detrainment rates of the bulk plume model.

[21] Although only the cloudy updrafts are considered here, the downdraft mass flux can be treated as a downward mapping in the same way. It may be necessary to account for the effects from, for example, precipitation driven downdrafts in deep convection and the subsidence shell in shallow convection [Heus and Jonker, 2007; Jonker et al., 2008]. Accounting for both the updrafts and downdrafts and the potential exchange between them in the mapping framework will be explored in future work.

[22] Acknowledgments. The authors thank two anonymous reviewers for their helpful reviews of an earlier version of this paper, and Marat Khairoutdinov for making the SAM model available. This research was 
partially supported by the DOE BER grant DE-FG02-08ER64556 as part of the ASR Program and NSF grants ATM-0754332 and AGS-1062016.

[23] The Editor thanks the anonymous reviewer for their assistance in evaluating this paper.

\section{References}

Arakawa, A., and W. H. Schubert (1974), Interaction of a cumulus cloud ensemble with the large-scale environment. Part I, J. Atmos. Sci., 31, 674-701.

Bretherton, C. S., J. R. McCaa, and H. Grenier (2004), A new parameterization for shallow cumulus convection and its application to marine subtropical cloud-topped boundary layers. Part I: Description and $1 \mathrm{D}$ results, Mon. Weather Rev., 132, 864-882.

Cheinet, S. (2004), A multiple mass flux parameterization for the surfacegenerated convection. Part II: Cloudy cores, J. Atmos. Sci., 61, 1093-1113.

Dawe, J. T., and P. H. Austin (2011a), Interpolation of LES cloud surfaces for use in direct calculations of entrainment and detrainment, Mon. Weather Rev., 139, 444-456.

Dawe, J. T., and P. H. Austin (2011b), The influence of the cloud shell on tracer budget measurements of LES cloud entrainment, J. Atmos. Sci., 68, 2909-2920.

Emanuel, K. A. (1991), A scheme for representing cumulus convection in large-scale models, J. Atmos. Sci., 48, 2313-2329.

Heus, T., and H. J. J. Jonker (2007), Subsiding shells around shallow cumulus clouds, J. Atmos. Sci., 65, 1003-1018.

Heus, T., G. van Dijk, H. J. J. Jonker, and H. E. A. van den Akker (2008), Mixing in shallow cumulus clouds studied by Lagrangian particle tracking, J. Atmos. Sci., 65, 2581-2597.

Holland, J. Z., and E. M. Rasmusson (1973), Measurement of atmospheric mass, energy, and momentum budgets over a 500-kilometer square of tropical ocean., Mon. Weather Rev., 101, 44-55.
Jonker, H. J. J., T. Heus, and P. P. Sullivan (2008), A refined view of vertical mass transport by cumulus convection, Geophys. Res. Lett., 35, L07810, doi:10.1029/2007GL032606.

Kuang, Z. (2010), Linear response functions of a cumulus ensemble to temperature and moisture perturbations and implication to the dynamics of convectively coupled waves, J. Atmos. Sci., 67, 941-962.

Kuang, Z., and C. S. Bretherton (2006), A mass-flux scheme view of a high-resolution simulation of a transition from shallow to deep cumulus convection, J. Atmos. Sci., 63, 1895-1909.

Neggers, R. A. J., A. P. Siebesma, and H. J. J. Jonker (2002), A multiparcel model for shallow cumulus convection., J. Atmos. Sci., 59, 1655-1668.

Nie, J., and Z. Kuang (2012), Responses of shallow cumulus convection to large-scale temperature and moisture perturbations: A comparison of large-eddy simulations and a convective parameterization based on stochastically entraining parcels, J. Atmos. Sci., 69, 1936-1956.

Romps, D. M. (2010), A direct measure of entrainment, J. Atmos. Sci., 67, 1908-1927.

Romps, D. M., and Z. Kuang (2010), Nature versus nurture in shallow convection, J. Atmos. Sci., 67, 1655-1666.

Siebesma, A. P., and J. W. M. Cuijpers (1995), Evaluation of parametric assumptions for shallow cumulus convection, J. Atmos. Sci., 52, 650-666.

Siebesma, A. P., et al. (2003), A large eddy simulation intercomparison study of shallow cumulus convection, J. Atmos. Sci., 60, 1201-1219.

Tulich, S. N., and B. E. Mapes (2010), Transient environmental sensitivities of explicitly simulated tropical convection, J. Atmos. Sci., 67, 923-940.

Yeo, K., and D. M. Romps (2012), Measurement of convective entrainment using Lagrangian particles, J. Atmos. Sci., in press.

Weil, J. C., P. P. Sullivan, and C. H. Moeng (2004), The use of large-eddy simulation in lagrangian particle dispersion models, J. Atmos. Sci., 61, 2877-2887. 